\title{
Individual Differences in Attention Allocation During a 2-dimensional Inhibitory Control Task
}

\author{
Emily R. Weichart \\ Department of Psychology \\ University of Virginia \\ Per B. Sederberg \\ Department of Psychology \\ University of Virginia
}

\begin{abstract}
Author Note
Correspondence concerning this article should be addressed to Per B. Sederberg, PhD., Department of Psychology, University of Virginia, Charlottesville, VA 22904. E-mail: pbs5u@virginia.edu
\end{abstract}




\begin{abstract}
It has been proposed that visual attention behaves as a spotlight or zoom lens that gradually focuses on goal-relevant features of a stimulus over the course of a trial. Several lines of evidence suggest that for spatially-contiguous stimuli, the spotlight naturally takes on the shape of a horizontally-biased ellipse. Analyses of group level behavior in the presence of horizontallyversus vertically-configured stimuli, however, potentially obfuscate an important source of between-subject variability in the early stages of attentional processing. In the current study, we used a 2-dimensional flanker task paradigm and nested variants of a model of within-trial attention and decision mechanisms to investigate individual differences in spotlight shapes. To account for the influence of distractor stimuli in both horizontal and vertical positions relative to the target, we operationalized the attentional spotlight as the density function for a bivariate normal distribution within our models. Horizontal and vertical shape parameters governing the spotlight were constrained to be equal in one model variant, and were allowed to vary in the other. Within-subject comparisons of Bayesian goodness of fit statistics revealed a general preference for an elliptical rather than a circular spotlight. Follow-up analyses, however, demonstrated substantial variability in spotlight shapes across subjects. Although data from most subjects were best-captured by a horizontally-biased elliptical spotlight, we observed individual differences in the extent of the bias, with some subjects even demonstrating a circular or vertically-biased elliptical spotlight.
\end{abstract}

Keywords: attention: theoretical and computational models; cognitive and attentional control 


\section{$1 \quad$ Introduction}

Although the mechanisms underlying visual attention remain a topic of active research, it is widely accepted that the focal area of attention can vary in size (Castiello \& Umiltà, 1992; C. Eriksen \& St James, 1986; Henderson, 1991; Jonides, 1983; LaBerge, 1983; Lavie, 1995) and that processing is more efficient when the focal area is small, compared to when it is large (C. Eriksen \& Schultz, 1979; C. Eriksen \& Yeh, 1985; Pan \& Eriksen, 1993; Umiltà, 1998). The Eriksen flanker task (B. Eriksen \& Eriksen, 1974), which requires participants to respond to a target while ignoring target-congruent or -incongruent distractor stimuli, has proven to be a particularly useful tool for studying attention. Notably, Gratton and colleagues (1988) used the flanker task to reveal a time-related component to attentional processing efficiency, such that incongruent distractors cause markedly less processing interference as response times (RTs) increase. These and other behavioral and electrophysiological findings suggest that distractor stimuli have an influence on processing early in the trial, but that it decreases through time (Burle et al., 2002; Czernochowski, 2015; Nigbur et al., 2015; Ridderinkhof, 2002). In their seminal theory, Eriksen and St. James (1986) provided the explanation that attention behaves as a zoom lens or shrinking spotlight that starts out wide and diffuse at the beginning of a trial and gradually focuses on the target.

The size and shape of the attentional spotlight has been extensively studied using mixtures of horizontally- and vertically-arranged flanker stimuli (Chen \& Tyler, 2002; Cohen \& Shoup, 1993; Livne \& Sagi, 2011; Vejnović \& Zdravković, 2015) and visual search paradigms (Hüttermann et al., 2014; Luck et al., 1989; Panagopoulos et al., 2004). By manipulating the 
spatial distance, position, and stimulus onset asynchrony of distractors relative to targets, for example, Pan and Eriksen (1993) concluded that the dimensions of the spotlight dynamically adjust from trial to trial based on the spatial configuration of the stimulus at hand. In line with these results, subsequent work showed that the spotlight can take on the shape of a ring (M. Müller \& Hübner, 2002), a Mexican hat (N. Müller et al., 2005) or can be divided among non-contiguous locations (Dubois et al., 2009; M. Müller et al., 2003; Treue \& Martinez-Trujillo, 2012) depending on the spatial arrangement of the stimuli and the demands of the task. Through group-level analyses of speed and accuracy, however, other studies have concluded that there is a dimensional bias to the spotlight, such that it is elliptical in shape, broadly distributed along the horizontal plane and narrowly distributed along the vertical plane (Andersen \& Kramer, 1993; Feng et al., 2007; Hüttermann et al., 2013). While we acknowledge that the notions of a stimulus-dependent spotlight and a horizontal attention bias are not mutually exclusive, we contend that the extent to which these processing features tradeoff within individual participants has not been thoroughly investigated.

In the current study, we used a 2-dimensional flanker task paradigm and a corresponding computational model to investigate the hypothesis that individuals vary in dimensional biases related to attentional allocation when controlling for the spatial configuration of stimuli across conditions. As shown in Figure 1, stimuli were designed to be identical in spatial distribution across conditions in an effort to limit stimulus-dependent modulation of the spotlight. Within each condition, we manipulated the arrangement of target-congruent and -incongruent distractors to assess behavioral biases. We fit two variants of a sequential sampling model of within-trial 
decision processing during the flanker task to each participant's data, which allowed us to calculate parameter estimates based on trial-level choices and RTs (Weichart et al., 2020). Both model variants contain an attentional spotlight, implemented as the density function for a bivariate normal distribution that narrows onto the target throughout the decision process. The shape of the spotlight is specified by separate horizontal and vertical standard deviation (SD) parameters. In the circular spotlight model, the shape parameters were constrained to be equal in order to reflect the horizontally- and vertically-symmetric spatial configuration of the stimuli. The alternative elliptical spotlight variant subsumes the circular spotlight model, and allows the horizontal and vertical shape parameters to take on different values to optimally fit each participant's data, if needed. Our results show that an elliptical rather than a circular spotlight is favored for most subjects, and demonstrate notable variability between subjects in terms of horizontal or vertical biases.

\section{Methods}

\subsection{Participants}

26 undergraduate students were recruited from the University of Virginia to participate in exchange for partial course credit. All participants provided informed consent in accordance with the requirements of the Institutional Review Board at the university.

\subsection{Stimuli and Apparatus}

A custom program using the State Machine Interface Library for Experiments (SMILE; https://github.com/compmem/smile) was written to present stimuli, track timing, and log 
responses. The experiment was administered on a desktop computer with Windows 10, connected to a 24-inch, 1920 x 1080-pixel LED display with a refresh rate of $120 \mathrm{~Hz}$. Stimuli were presented in white text on a dark gray background. Participants made responses using the outer two keys of a four-key Black Box ToolKit response pad. Stimulus arrays were comprised of 13 left- or right-facing arrows arranged in a diamond formation. Distractor arrows took on one of five configurations, as illustrated in Figure 1, and participants were asked to indicate the direction of the arrow in the center of the array while ignoring all distractors. The stimuli were designed in consideration of research demonstrating that distractor interference is positively correlated with proximity of distractors to target (Pan \& Eriksen, 1993; Andersen \& Kramer, 1993; Feng, Jiang, \& He, 2007). In the easy condition, all distractors were congruent to the target. In the moderate and hard conditions, distractors were incongruent to the target in the outer and inner layers of the array, respectively. Horizontal and vertical conditions were included to test for asymmetries in dimension-level response competition. On each trial, the array was presented in one of eight locations around the screen. Possible locations were equidistant from the center of the screen in increments of 45 degrees. Task condition (easy, moderate, hard, horizontal, or vertical), target direction (left or right) and screen location ( 0,45 , $90,135,180,225,270$, or 315 degrees) were counterbalanced and randomized within-block. 


$\begin{array}{ccccc}\text { Easy } & \text { Moderate } & \text { Hard } & \text { Horizontal } & \text { Vertical } \\ > & < & > & > & < \\ >>> & <>< & ><> & <<< & <>< \\ >>>>> & <>>< & ><><> & <>>>< & ><><> \\ >>> & <>< & ><> & <<< & <>< \\ > & < & > & > & <\end{array}$

Figure 1: Examples of stimuli. Colors are used to highlight the contrasting directions configurations of left (red) and right (blue) arrows. All stimuli shown here contain a left-facing target, but analogous stimuli with right-facing targets were included in the experiment as well.

\subsection{Procedure}

Participants provided informed consent and were seated in individual testing rooms. Instructions and example stimuli appeared on the screen, and instructions were also provided verbally by an experimenter. A practice module with visual feedback for correct and incorrect responses was administered until the experimenter verified that the participant understood the task ( $\sim 1$ minute). Prior to beginning the main task, the experimenter provided the following information: "You will complete eight blocks of the task, each lasting about 2 minutes. At the end of each block, you will receive a score based on speed and accuracy. Please try to get the highest score that you can." Once the task began, a fixation cross appeared in the center of the screen and remained for the duration of the block. Stimuli appeared on the screen until a response was made, or for a maximum of $3000 \mathrm{~ms}$. Participants responded by pressing the leftmost key on the response pad if the arrow in the center of the array pointed left, and the rightmost key if the center arrow pointed right. Only responses made at least $150 \mathrm{~ms}$ after the stimulus appeared were recorded. At the end 
of each block, participants received a numerical score between 0 and 100 that was calculated as shown in Equations 1-3:

$$
\begin{gathered}
\text { accuracy }=\frac{\frac{N_{\text {correct }}}{N_{\text {total }}}-0.5}{0.5} \\
\text { speed }=\frac{\sum_{i \in I} \frac{\log \left(R T_{\max }+1.0\right)-\log (i+1.0)}{\log \left(R T_{\max }+1.0\right)-\log \left(R T_{\min }+1.0\right)}}{N_{\text {total }}} \\
\text { score }=\text { speed } * \text { accuracy } * 100
\end{gathered}
$$

where $I$ is a vector of RTs in seconds. Within this scoring metric, performance across conditions was scaled between chance (0.5) and perfect accuracy (1.0), and RTs were scaled to fall within an expected range of $R T_{\text {min }}=350 \mathrm{~ms}$ to $R T_{\max }=1350 \mathrm{~ms}$. Log transforms were used in Equation 2 to correct for rightward skew in the RT distributions, and 1.0 was added to prevent against negative $\log$ RT values. To earn a high score, participants needed to respond both quickly and accurately in all conditions. Across 8 blocks each consisting of 80 trials, participants completed a total of 640 trials. 


\subsection{Computational Models}

The base model in our current investigation was designed after the zoom lens theory of Eriksen and St. James (1986), with decision and attention mechanisms implemented within a leaky-competing accumulator (LCA; Usher \& McClelland, 2001) model framework. Specifically, we modified the LCA-control model of the flanker task described by Weichart and colleagues (2020) to accomodate 2-dimensional stimuli. Details of the LCA-control model are provided in the original article, but will be summarized here. In LCA-control and other accumulator models, trial-level decisions are thought to result from the noisy build-up of evidence for competing response options up to a threshold $(\alpha)$. Evidence accumulation is governed by drift rates that reflect the strength of information provided by the stimulus, lateral inhibition $(\beta)$, and passive decay through time $(\kappa)$. Each accumulator $i$ with drift rate $\rho_{i}$ and current evidence $x_{i}$ is updated continuously as shown in Equation 4.

$$
\begin{gathered}
d x_{i}=\left(\rho_{i}-\kappa x_{i}-\beta \sum_{j \neq i} x_{j}\right) \frac{d t}{\Delta t}+\xi \sqrt{\frac{d t}{\Delta t}} \\
x_{i} \rightarrow \max \left(x_{i}, 0\right)
\end{gathered}
$$

Time was discretized via the Euler method, using a step size of $d t=0.01$ modified by a time constant of $\Delta_{t}=0.1$. The degree of noise is represented as a driftless Wiener process distributed as $\xi \sim \mathcal{N}(0,1)$. Responses are made in favor of the first accumulator to exceed $\alpha$, and RTs are equal to the duration of the decision process plus a nondecision time parameter $(\tau)$. Similar to the shrinking spotlight model designed by White, Ratcliff, and Starns (SSP; 2011), 
LCA-control features an attentional spotlight that gradually focuses on the target throughout the trial, but, unlike the original SSP, the spotlight shrinks due to the dynamics of the decision process and not simply time. For our current purposes, the spotlight takes the form of a density function for a bivariate normal distribution centered on the target stimulus with initial horizontal and vertical SDs of $s d_{0}(h)$ and $s d_{0}(v)$, respectively. The spotlight shrinks as a function of an online measure of cognitive control $(c)$, modified by a rate parameter $\left(r_{d}\right)$ as shown in Equations 5 and 6.

$$
\begin{aligned}
& s d_{a}(h)=s d_{0}(h)-r_{d} c \\
& s d_{a}(v)=s d_{0}(v)-r_{d} c
\end{aligned}
$$

A ratio parameter $\Theta$ governs the relationship between $s d_{0}(h)$ and $s d_{0}(v)$, such that $\Theta=\frac{s d_{0}(v)}{s d_{0}(h)} . \Theta$ was fixed to 1.0 when fitting the circular spotlight model, but was a free parameter when fitting the elliptical spotlight model. The behaviors of the attentional spotlights in the circular and elliptical spotlight models are illustrated in Figure 2. 


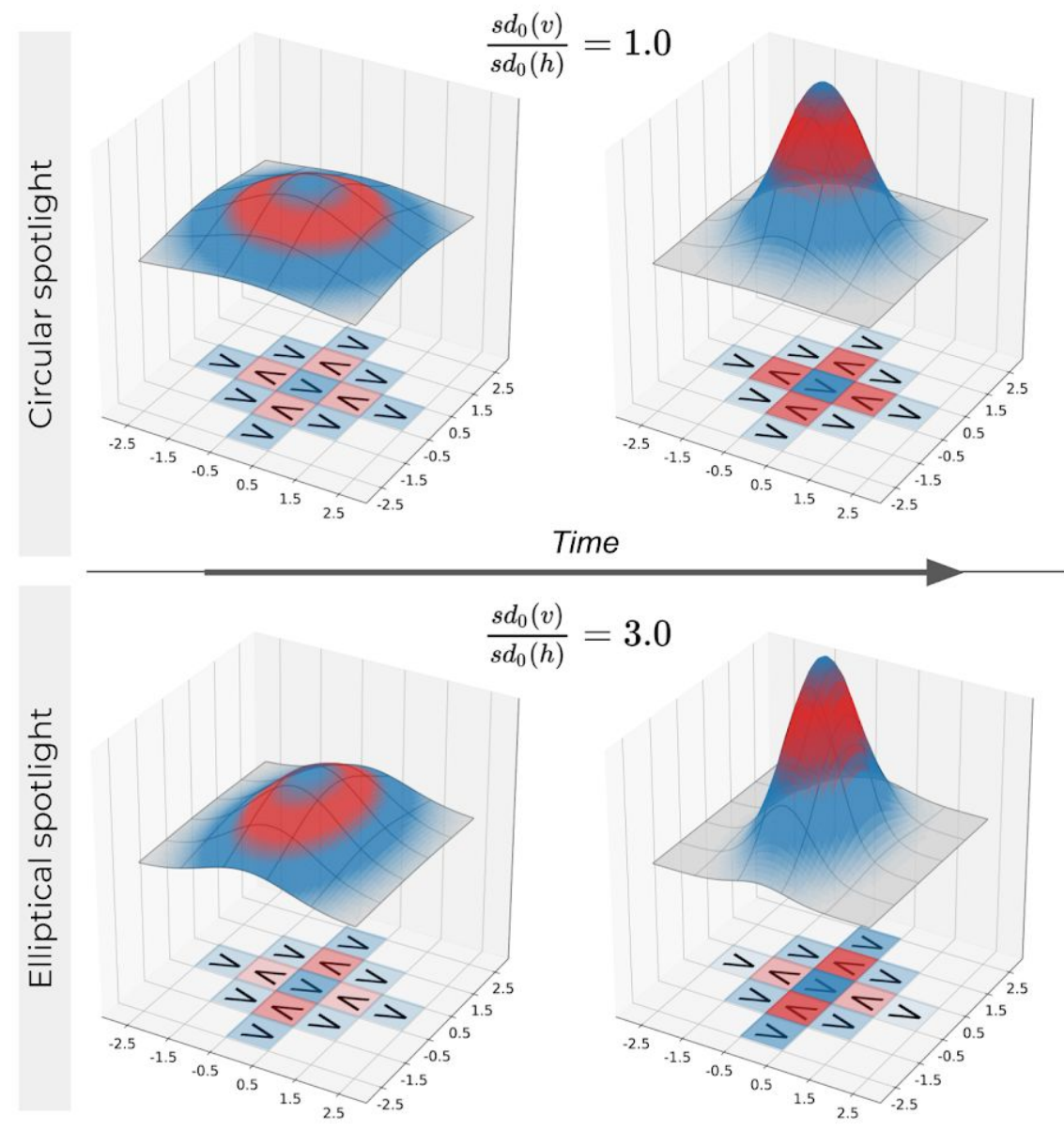

Figure 2: Representation of the shrinking 2-dimensional spotlight of visual attention. Over the course of a trial, illustrated from left to right, the spotlight shrinks and focuses on the center arrow of a stimulus array, rendered in each subplot at $\mathrm{z}=0$. The strength of visual attention allocated to each arrow in a stimulus array is calculated from the density of a bivariate normal distribution within the corresponding unit square. The top panels show a circular spotlight, such that the shape parameters of the bivariate normal are constrained to be equal. The bottom panels show an elliptical spotlight, such that the two shape parameters are free to vary.

The mechanism for cognitive control is based on descriptions of reactive control discussed in Braver's dual mechanisms of control framework (Braver, 2012; Braver et al., 2008; De Pisapia 
\& Braver, 2006), and is calculated as the cumulative distance between total evidence and a conflict resolution threshold, $\delta$. The continuous change in $c$ is given by Equation 7.

$$
d c=\left(\delta-\sum_{i \in\{1,2\}} x_{i}\right) \frac{d t}{\Delta_{t}}
$$

Each individual arrow in a stimulus array occupies one square unit of perceptual space, and the spotlight is centered on the target. Drift rates corresponding to correct $\left(\rho_{1}\right)$ and incorrect $\left(\rho_{2}\right)$ responses are calculated as the total volume of the spotlight allocated to target-congruent and target-incongruent arrows, respectively. Within each unit square of the 5-by-5 stimulus array, we used the trapezoidal method to estimate the bivariate probability density at 100 equally-spaced points (Kalambet et al., 2018). The spotlight volume allocated to each unit square was then estimated from the integral of the density values over the range of interest, multiplied by the perceptual strength of a single arrow $(p)$ as shown in Equation 8.

$$
V=p \int_{n}^{n+1} \int_{m}^{m+1} \frac{1}{2 \pi s d_{a}(h) s d_{a}(v)} \exp \left(-\frac{1}{2}\left[\frac{x^{2}}{s d_{a}(h)^{2}}+\frac{y^{2}}{s d_{a}(v)^{2}}\right]\right) d y d x
$$

Values of $d x$ and $d y$ were set to 0.1 , and $(x, y)$ coordinates fell within the unit square occupied by an arrow with vertices $(n, m),(n+1, m),(n+1, m+1),(n, m+1)$, such that $n, m \in[-2.5,-1.5,-0.5,0.5,1.5]$. The conceptualization of arrow stimuli within a coordinate place is illustrated in Figure 2. A summary of free parameters in our two models and their respective prior distributions are shown in Table 1. 
Table 1: Model free parameters and priors

\begin{tabular}{|c|l|}
\hline Parameter Description & \multicolumn{1}{|c|}{ Prior } \\
\hline Decision threshold & $\operatorname{logit}\left(\frac{\alpha}{30.0}\right) \sim \mathcal{N}(\mu=-1.0, \sigma=1.2)$ \\
\hline Lateral inhibition & $\operatorname{logit}(\beta) \sim \mathcal{N}(\mu=0.0, \sigma=1.4)$ \\
\hline Evidence leak & $\operatorname{logit}(\kappa) \sim \mathcal{N}(\mu=0.0, \sigma=1.4)$ \\
\hline Nondecision time & $\operatorname{logit}\left(\frac{\tau}{R T_{\text {min }}}\right) \sim \mathcal{N}(\mu=-0.2, \sigma=1.2)$ \\
\hline Initial spotlight SD (horizontal) & $\operatorname{logit}\left(\frac{s d_{0}(h)-0.1}{30.0-0.1}\right) \sim \mathcal{N}(\mu=-0.2, \sigma=1.2)$ \\
\hline Spotlight dimension ratio ${ }^{1}$ & $\Theta \sim \gamma(\alpha=3.0, \beta=2.0)$ \\
\hline Rate of focus & $\operatorname{logit}\left(\frac{r_{d}}{20.0}\right) \sim \mathcal{N}(\mu=-2.0, \sigma=1.0)$ \\
\hline Conflict resolution threshold & $\operatorname{logit}\left(\frac{\delta}{30.0}\right) \sim \mathcal{N}(\mu=-1.0, \sigma=1.2)$ \\
\hline Perceptual input strength & $\operatorname{logit}\left(\frac{p}{20.0}\right) \sim \mathcal{N}(\mu=-0.8, \sigma=1.2)$ \\
\hline
\end{tabular}

\subsection{Model Fitting and Assessment}

We used the model-fitting procedures described in detail by Weichart and colleagues (2020) with probability density approximation methods (PDA; Turner \& Sederberg, 2014) that were implemented via custom programs in RunDEMC (https://github.com/compmem/RunDEMC). Broadly, fitting a model to a set of trial-level data from an individual subject was a 6-step

\footnotetext{
${ }^{1}$ Spotlight dimension ratio was a free parameter in the elliptical spotlight model only.
} 
process: First, we specified the relevant model as a system of equations, prior distributions that were determined through a series of pilot investigations, and starting values for each free parameter. Next, the model was simulated 30,000 times using the starting set of parameter values. This step generated distributions of data in each task condition. The probability density function of the simulated data was then approximated using an Epanechnikov kernel (Turner \& Sederberg, 2014; Turner et al., 2016). The estimated functional form of the simulated data approximates the likelihood function of each observed response under the current set of parameter values. The posterior density of the parameter set was calculated as a combination of the likelihood function and the priors. A new proposal parameter set was then selected using differential evolution with Markov chain Monte Carlo (DE-MCMC; Ter Braak, 2006; Turner \& Sederberg, 2012; Turner et al., 2013), a genetic algorithm that selects parameter values according to the success of previous proposals. This procedure was implemented for 800 iterations across 90 chains. Each model's goodness of fit was assessed via Bayesian information criterion (BIC), which considers both the log likelihood of the best-fitting parameters (maximum log likelihood; MLL) and model parsimony (number of free parameters). BIC was calculated using Equation 9

$$
B I C=\ln (n) k-2 \ln (M L L)
$$

where $n$ is the number of data points and $k$ is the number of free parameters in the model of interest (Claeskens \& Hjort, 2001; Wit et al., 2012). BIC values for the circular and elliptical spotlight models were compared within-subject, with lower BIC values indicating a better fit. 


\section{$3 \quad$ Results}

\subsection{Behavioral Results}

Performance scores based on speed and accuracy were calculated for each participant and task condition using Equations 1-3. As shown in the left panel of Figure 3, we observed the expected pattern of decreasing performance from the easy to the moderate condition $(t(25)=9.65, p<0.0001)$ and from the moderate to the hard condition $(t(25)=8.74, p<0.0001)$. Performance was also better across participants in the horizontal compared to the vertical condition $(t(25)=3.92, p<0.001)$. The right panel of Figure 3 presents nuanced insight into the latter comparison. The majority (19 out of 26) of participants performed better in the horizontal compared to the vertical condition, but some participants ( 5 out of 26) displayed the opposite pattern of results. This indicates that most participants were better at ignoring incongruent distractors that were placed immediately above and below the target, compared to those that were placed immediately to the left and right. Model predictions shown in the left panel of Figure 3 will be discussed in the Model Results section below. 

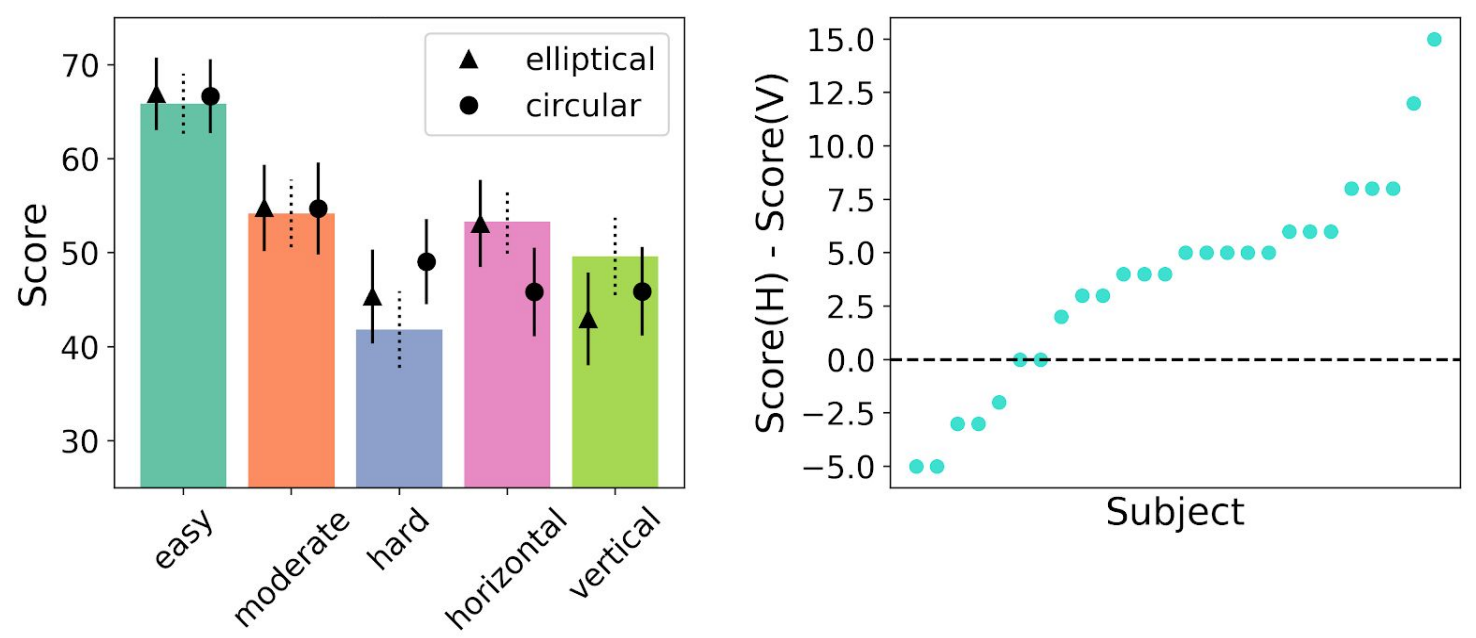

Figure 3: Behavioral results. Left Panel: Observed and model-predicted scores within condition. Bars show observed mean scores. Triangular and circular points show mean scores predicted by the elliptical and circular spotlight models, respectively. Error bars show 95\% confidence intervals of scores across subjects. Right Panel: Score difference, horizontal vs. vertical conditions. Differences in task scores in the horizontal and vertical task conditions, calculated within-subject.

\subsection{Model Results}

Before assessing our results, we first compared MLL values from the circular and elliptical spotlight models at the level of each subject as a check for our fitting procedures. Because the elliptical model subsumes the circular variant, the MLL for the elliptical model should be greater than or approximately equal to that of the circular model in all cases. We indeed found this to be true for all subjects. We then calculated differences in BIC values between the two models in order to account for model complexity in addition to MLL when assessing model performance. Results are shown in Figure 4. BIC values for the circular model exceed those of the elliptical model in cases where the addition of the free parameter $\Theta$ did not meaningfully improve the fit 
of the model ( 6 out of 26 subjects). For 20 out of 26 subjects, however, the additional flexibility of the elliptical model provided meaningfully improved model fits as determined by BIC.

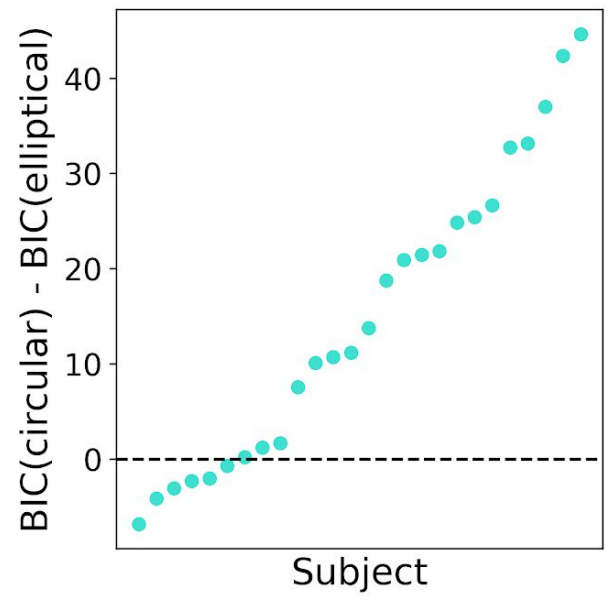

Figure 4: Subject-level differences in BIC values between the circular spotlight and elliptical spotlight models. The elliptical model outperforms the circular model for the majority of subjects (lower BIC values indicate better fit).

We next wanted to observe the range of spotlight dimensions calculated within the elliptical model. We first determined the scaled difference between horizontal and vertical standard deviations $\left(D_{h, v}\right)$ from each subject's best-fitting parameter values in the elliptical spotlight model using Equation 10:

$$
D_{h, v}=\frac{s d_{0}(h)-s d_{0}(v)}{\frac{s d_{0}(h)+s d_{0}(v)}{2}}
$$

where $s d_{0}(v)=s d_{0}(h) \Theta$. The left panel of Figure 5 shows the range of best-fitting spotlight shapes for each subject: data from 17 out of 26 subjects were best-fit by a horizontally-biased spotlight (points above $\mathrm{y}=0.0$ ), and data from 9 out of 26 subjects were best-fit by a 
vertically-biased spotlight (points below $\mathrm{y}=0.0$ ). As shown by the right panel of Figure 5, we identified a positive correlation between the extent of horizontal bias in spotlight shape and the extent of performance benefit in the horizontal relative to the vertical task condition $\left(R^{2}=0.31\right)$. The direction of this relationship indicates that participants with a horizontally-biased attentional spotlight are better equipped to ignore distractors placed above and below the target compared to those placed to the left and right of the target, and participants with a vertically-biased spotlight show the opposite performance benefit.
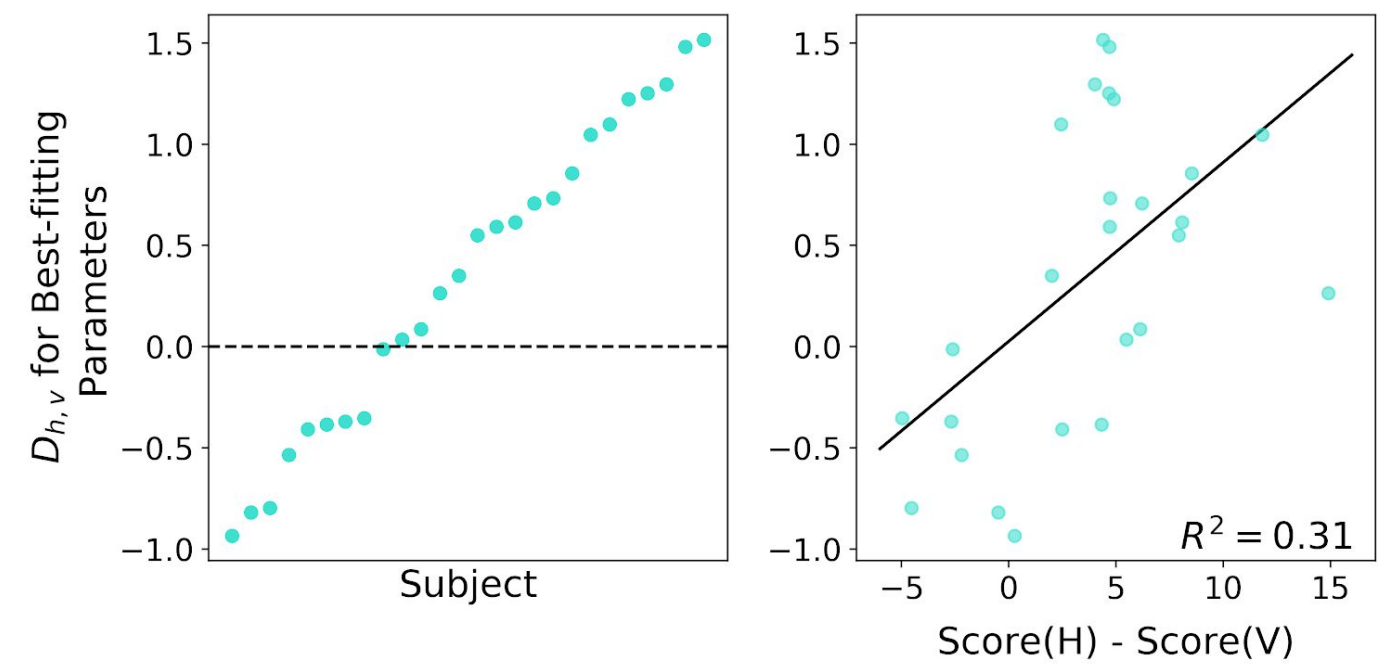

Figure 5: Model Results. Left Panel: Subject-level scaled differences in best-fitting horizontal and vertical shape parameters for the spotlight in the elliptical spotlight model. Right Panel: Behavior vs. parameter estimates.

Spotlight shape asymmetry predicts behavioral performance in the horizontal and vertical conditions.

To investigate why the elliptical model tended to fit better than the circular variant, we simulated data in each model, and compared it to the behavior that our participants actually produced. We first found best-fitting parameters for each model and subject by identifying the particle in the 
joint posterior with the maximum weight. Using best-fitting parameters and the relevant model, we generated 10,000 choice-RT trials in each condition. We were then able to calculate a performance score for each model and subject using Equations 1-3. Ranges of condition-level scores generated by each model are shown in the left panel of Figure 3, alongside the observed data. Figure 3 shows that both models predict the observed pattern of decreasing performance as we move from the easy, to the moderate, to the hard conditions. The elliptical model also captures the observed pattern of better performance in the moderate and horizontal conditions, compared to the hard and vertical conditions. Given that data from most participants favored a horizontally-biased spotlight (see Figure 5), elliptical model predictions reflect the fact that moderate and horizontal stimuli contained the identical configuration of distractors along the horizontal midline, as did the hard and vertical conditions (see Figure 1). While the elliptical model made accurate predictions in the moderate and horizontal conditions, it was unable to capture the pattern of better performance in the vertical condition compared to the hard condition. We suspect this is due to perceptual continuity or grouping effects that are not currently accounted for in the model, but that would disproportionately affect performance in the hard condition due to the deliberately "interrupted" configuration of the distractors in the array (Livne \& Sagi, 2010; Manassi et al., 2012). Problems arising from a lack of perceptual continuity-related mechanisms appear to be exacerbated by over-constraint of the spotlight dimensions in the circular model. In addition to overestimating performance in the hard condition, the circular model underestimates performance in both the horizontal and vertical conditions. Because the spotlight in the circular model is constrained to be round, it is unable to 
predict the observed differences in performance between the horizontal and vertical conditions that are shown in the right panel of Figure 3.

\section{Discussion}

Here, we used model-based analyses to investigate individual differences in the shape of the attended visual area while subjects responded to two-dimensional flanker task stimuli. Given evidence of an attentional spotlight that dynamically adjusts to the spatial configuration of stimuli from trial to trial (Pan \& Eriksen, 1993), we developed stimulus arrays that were perceptually identical across conditions in order to identify subject-level biases in spotlight dimensions. We constructed two variants of a model that was designed after the zoom lens hypothesis of visual attention (C. Eriksen \& St James, 1986; Weichart et al., 2020). Consistent with previous results (Andersen \& Kramer, 1993; Feng et al., 2007; Hüttermann et al., 2013), we found that most participants use an elliptical spotlight, specifically one with a horizontal bias. This was not the case for all subjects, however, and we identified a widely variable range of spotlight configurations.

Previous studies investigating dimensional biases in visual attention have inferred the shape of the spotlight based on group-level differences in responses to horizontally- and vertically-configured stimuli. To explore individual differences in attentional biases, our model-based methods allowed us to account for the spotlight configuration's critical effects on the cascade of mechanisms that ultimately results in a specific pattern of behavior. Our results therefore add to the existing literature on attentional allocation, highlighting individual 
differences in dimensional biases that should be considered in future work. For example, the models in the current study relegated behavioral differences between the horizontal and vertical conditions to differences in spotlight dimensions captured by values of $s d_{0}(h)$ and $s d_{0}(v)$. Follow-up work will investigate whether splitting the $r_{d}$ parameter into separate horizontal and vertical values will further improve model fits, which would indicate that focusing attentional resources occurs at different rates between the two dimensions. Regardless of the precise mechanisms involved, we have presented conclusive evidence of dimension-dependent differences in attentional processes that have not been considered in existing mechanistic models of the flanker task. All other within-trial flanker decision models have been designed to fit data from stimulus arrays oriented along a single horizontal plane (Hübner et al., 2010; Ulrich et al., 2015; White et al., 2011). Real-world challenges to visual attention, however, require integrated processing across multiple spatial dimensions. By failing to consider multidimensional stimuli, our results indicate that these models are potentially missing an important source of variability between individuals in visual processing mechanisms.

Open Practices Statement: The data, stimuli, and experiment code used in the current study are available via the Center for Open Science at https://osf.io/nef6j/ (DOI:

10.17605/OSF.IO/NEF6J). 


\section{References}

Andersen, G., \& Kramer, A. (1993). Limits of focused attention in three-dimensional space. Perception \& Psychophysics, 53(6), 658-667.

Braver, T. (2012). The variable nature of cognitive control: a dual mechanisms framework. Trends in Cognitive Sciences, 16(2), 106-113.

Braver, T., Gray, J., \& Burgess, G. (2008). Explaining the Many Varieties of Working Memory Variation: Dual Mechanisms of Cognitive Control. Variation in Working Memory, 76-106.

Burle, B., Possamaï, C.-A., Vidal, F., Bonnet, M., \& Hasbroucq, T. (2002). Executive control in the Simon effect: an electromyographic and distributional analysis. Psychological Research, 66(4), 324-336.

Castiello, U., \& Umiltà, F. (1992). Splitting focal attention. Journal of Experimental Psychology. Human Perception and Performance, 18(3), 837-848.

Chen, C.-C., \& Tyler, C. (2002). Lateral modulation of contrast discrimination: flanker orientation effects. Journal of Vision, 2(6), 520-530.

Claeskens, G., \& Hjort, N.-L. (2001). Model Selection and Model Averaging. Cambridge: Cambridge University Press.

Cohen, A., \& Shoup, R. (1993). Orientation asymmetry in the flanker task. Perception \& Psychophysics, 53(6), 693-703.

Czernochowski, D. (2015). ERPs dissociate proactive and reactive control: evidence from a task-switching paradigm with informative and uninformative cues. Cognitive, Affective \& Behavioral Neuroscience, 15(1), 117-131.

De Pisapia, N., \& Braver, T. (2006). A model of dual control mechanisms through anterior 
cingulate and prefrontal cortex interactions. Neurocomputing, 69(10-12), 1322-1326.

Dubois, J., Hamker, F., \& VanRullen, R. (2009). Attentional selection of noncontiguous locations: the spotlight is only transiently "split." Journal of Vision, 9(5), 3.1-11.

Eriksen, B., \& Eriksen, C. (1974). Effects of noise letters upon the identification of a target letter in a nonsearch task. Perception \& Psychophysics, 16(1), 143-149.

Eriksen, C., \& Schultz, D. (1979). Information processing in visual search: a continuous flow conception and experimental results. Perception \& Psychophysics, 25(4), 249-263.

Eriksen, C., \& St James, J. (1986). Visual attention within and around the field of focal attention: a zoom lens model. Perception \& Psychophysics, 40(4), 225-240.

Eriksen, C., \& Yeh, Y. (1985). Allocation of attention in the visual field. Journal of Experimental Psychology. Human Perception and Performance, 11(5), 583-597.

Feng, C., Jiang, Y., \& He, S. (2007). Horizontal and vertical asymmetry in visual spatial crowding effects. Journal of Vision, 7(2), 13.

Gratton, G., Coles, M., Sirevaag, E., Eriksen, C., \& Donchin, E. (1988). Pre- and poststimulus activation of response channels: a psychophysiological analysis. Journal of Experimental Psychology. Human Perception and Performance, 14(3), 331-344.

Henderson, J. (1991). Stimulus discrimination following covert attentional orienting to an exogenous cue. Journal of Experimental Psychology. Human Perception and Performance, 17(1), 91-106.

Hübner, R., Steinhauser, M., \& Lehle, C. (2010). A dual-stage two-phase model of selective attention. Psychological Review, 117(3), 759-784.

Hüttermann, S., Memmert, D., \& Simons, D. (2014). The size and shape of the attentional 
"spotlight" varies with differences in sports expertise. Journal of Experimental Psychology: Applied, 20(2), 147-157.

Hüttermann, S., Memmert, D., Simons, D., \& Bock, O. (2013). Fixation strategy influences the ability to focus attention on two spatially separate objects. PloS One, 8(6), e65673.

Jonides, J. (1983). Further toward a model of the Mind's eye's movement. Bulletin of the Psychonomic Society, 21(4), 247-250.

Kalambet, Y., Kozmin, Y., \& Samokhin, A. (2018). Comparison of integration rules in the case of very narrow chromatographic peaks. In Chemometrics and Intelligent Laboratory Systems (Vol. 179, pp. 22-30). https://doi.org/10.1016/j.chemolab.2018.06.001

LaBerge, D. (1983). Spatial extent of attention to letters and words. Journal of Experimental Psychology: Human Perception and Performance, 9(3), 371-379.

Lavie, N. (1995). Perceptual load as a necessary condition for selective attention. Journal of Experimental Psychology: Human Perception and Performance, 21(3), 451-468.

Livne, T., \& Sagi, D. (2010). How do flankers' relations affect crowding? Journal of Vision, 10(3), 1.1-14.

Livne, T., \& Sagi, D. (2011). Multiple levels of orientation anisotropy in crowding with Gabor flankers. Journal of Vision, 11(13), 18.

Luck, S., Hillyard, S., Mangun, G., \& Gazzaniga, M. (1989). Independent hemispheric attentional systems mediate visual search in split-brain patients. Nature, 342(6249), $543-545$.

Manassi, M., Sayim, B., \& Herzog, H. (2012). Grouping, pooling, and when bigger is better in visual crowding. Journal of Vision, 12(10), 13. 
Müller, M., \& Hübner, R. (2002). Can the spotlight of attention be shaped like a doughnut? Evidence from steady-state visual evoked potentials. Psychological Science, 13(2), 119-124.

Müller, M., Malinowski, P., Gruber, T., \& Hillyard, S. (2003). Sustained division of the attentional spotlight. Nature, 424(6946), 309-312.

Müller, N., Mollenhauer, M., Rösler, A., \& Kleinschmidt, A. (2005). The attentional field has a Mexican hat distribution. Vision Research, 45(9), 1129-1137.

Nigbur, R., Schneider, J., Sommer, W., Dimigen, O., \& Stürmer, B. (2015). Ad-hoc and context-dependent adjustments of selective attention in conflict control: an ERP study with visual probes. NeuroImage, 107, 76-84.

Panagopoulos, A., Von Grünau, M., \& Galera, C. (2004). Attentive mechanisms in visual search. Spatial Vision, 17(4-5), 353-371.

Pan, K., \& Eriksen, C. W. (1993). Attentional distribution in the visual field during same-different judgments as assessed by response competition. Perception $\&$ Psychophysics, 53(2), 134-144.

Ridderinkhof, K. (2002). Micro- and macro-adjustments of task set: activation and suppression in conflict tasks. Psychological Research, 66(4), 312-323.

Ter Braak, C. (2006). A Markov Chain Monte Carlo version of the genetic algorithm Differential Evolution: easy Bayesian computing for real parameter spaces. Statistics and Computing, $16(3), 239-249$.

Treue, S., \& Martinez-Trujillo, J. (2012). The spotlight of attention: shifting, resizing and splitting receptive fields when processing visual motion. E-Neuroforum, 18(3). 
https://doi.org/10.1007/s13295-012-0034-9

Turner, B., \& Sederberg, P. (2012). Approximate Bayesian computation with differential evolution. Journal of Mathematical Psychology, 56(5), 375-385.

Turner, B., \& Sederberg, P. (2014). A generalized, likelihood-free method for posterior estimation. Psychonomic Bulletin \& Review, 21(2), 227-250.

Turner, B., Sederberg, P., Brown, S., \& Steyvers, M. (2013). A method for efficiently sampling from distributions with correlated dimensions. Psychological Methods, 18(3), 368-384.

Turner, B., Sederberg, P., \& McClelland, J. (2016). Bayesian analysis of simulation-based models. Journal of Mathematical Psychology, 72, 191-199.

Ulrich, R., Schröter, H., Leuthold, H., \& Birngruber, T. (2015). Automatic and controlled stimulus processing in conflict tasks: Superimposed diffusion processes and delta functions. In Cognitive Psychology (Vol. 78, pp. 148-174). https://doi.org/10.1016/j.cogpsych.2015.02.005

Umiltà, F. (1998). The Control of the Attentional Focus. European Journal of Cognitive Psychology, 10(3), 225-246.

Usher, M., \& McClelland, J. (2001). The time course of perceptual choice: the leaky, competing accumulator model. Psychological Review, 108(3), 550-592.

Vejnović, D., \& Zdravković, S. (2015). Side flankers produce less crowding, but only for letters. Cognition, 143, 217-227.

Weichart, E., Turner, B., \& Sederberg, P. (2020). A model of dynamic, within-trial conflict resolution for decision making. Psychological Review. https://doi.org/10.31234/osf.io/2hk8t

White, C., Ratcliff, R., \& Starns, J. (2011). Diffusion models of the flanker task: discrete versus 
gradual attentional selection. Cognitive Psychology, 63(4), 210-238.

Wit, E., van den Heuvel, E., \& Romeijn, J.-W. (2012). “All models are wrong...”: an introduction to model uncertainty. Statistica Neerlandica, 66(3), 217-236. 